\title{
Numerical investigation of snowstorm filling
}

\author{
Ferenc Safranyik ${ }^{1 *}$, Attila Varga $^{2}$ \\ ${ }^{1}$ Eötvös Loránd University, Faculty of Informatics, Savaria Institute of Technology, 9700 Szombathely, Hungary \\ ${ }^{2}$ Knorr-Bremse Rail Systems Budapest, 1238, Budapest, Hungary
}

\begin{abstract}
During industrial air preparation, one of the most important issues is the proper moisture content. In vehicle brake systems, compressed air is commonly used, where condensate in the system can lead to serious problems as corrosion, frosting, etc. For avoiding malfunctions, the relative humidity of the media must decrease to $20-40 \%$, by adsorbing its moisture content. To achieve this, air dryer units filled with desiccant granular media is commonly used in railway industry. The compressed air flows through the dryer, thus the particles can bind the water vapour. If the packing density of the desiccant can increase, the efficiency of the dryer can improve. In this paper an interesting method for container filling, the snowstorm technique was investigated and optimized by applying discrete element method. Essence of the device is a rod system placed below the filler bin, which ensures even distribution of the particles. By the numerical analysis it was revealed, the particles' lateral velocity is increased because of the snowstorm system, but with non-optimal arrangement, the particle flow can be significantly blocked. Based on our systematic analysis, optimal geometry of the particle distribution system was found, which ensures settling more particles by $9.5 \%$ into the dryer reservoir.
\end{abstract}

\section{Introduction}

Industrial air brake malfunctions are commonly caused by moisture and oil pollution. Water vapour appearing in pneumatic break system of vehicles may worse efficiency due to corrosion or frostbite, therefore preparation of compressed air has great importance in vehicle industry. Most of the problems can be effectively prevent, by applying dryers for reducing humidity of the air system. Reservoirs filled with porous, desiccant media are frequently utilized for decreasing moisture content of the industrial air. The compressed air is blown through the reservoir filled with spherical particles for reducing its moisture content. Sandstone is one of the commonly used adsorbents.

Efficiency of the dryer unit depends on two main factors: on chemical properties of the desiccant and on packing density of the particles filled into the dryer. In industry, vibratory shakers are commonly used for in-

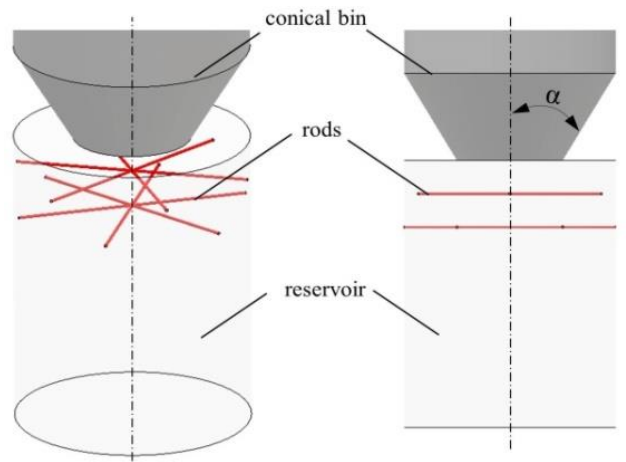

Fig. 1. Main components of the snowstorm filler creasing mass of the material loaded into the reservoir, however in this way energy consumption of the filling procedure is considerable grow. When the necessary packing density could be achieved without applying external excitation, the efficiency of drying may increase. Moreover, operation and maintenance of the reservoir filling can be also simplified.

The so called snowstorm filling technique, patented by Harris in 1964 [1] is a very promising method for optimizing the filling process. The main idea of the technique is using multiple obstacles (radially placed rods or mesh) above the reservoir to evenly distribute the particles falling through the device (Fig. 1). The procedure can be separated into two physical phenomena: material discharge from a conical bin, and interaction of distributing elements with the particle flow (in our case, rod shaped obstacles were used). Currently the device is applied in several fields of industry, however working principles of it is not carefully investigated, as geometrical configuration of the snowstorm device used in a certain technological process was determined based on observations until this time. Main aim of this work is evaluating the particle flow in the snowstorm device for optimizing its geometry, by applying discrete element method (DEM).

\subsection{Particle discharge}

Outflow of conical bins is well-known based on previous researches $[2,3]$. The outflow velocity of particles is independent from the filling level of the container in steady state. Based on the mechanical

* Corresponding author: $\underline{\text { sf@inf.elte.hu }}$

A video is available at https://doi.org/10.48448/g96d-qp50 
behaviour of the stored material and the geometrical parameters of the container (mostly on cone half angle, designated with $\alpha$ in Fig. 1), special flow patterns can be formed. In case of steep bins, when the material filled first, flow out first and during the discharge the whole bulk is in motion, we can speak about mass flow. When the cone half angle is larger, stagnant zones are forming on the bin's wall and the bulk flows only in a narrow channel above the outlet, which is the funnel flow discharge. Outflow rate of cohesionless particulates can predict both analytically $[4,5]$ and numerically $[2,3,6]$. In this study the numerical approach was used by applying DEM.

\subsection{Particle packing}

Minimizing the space occupied by the particles in a certain volume can be essential for improving storage and transport of granular materials. Therefore, container filling with particulate matter has long been a topic of interest for scholars, as it is a significant procedure both in industrial and in everyday life. Experimental measurements made from 1960 s revealed that, random packing of spheres can be classified into two welldefined types. Gravitational deposition of spherical particles leads to random loose packing, but if the reservoir is gently shaken, the random dense state forms [7]. Based on Scott's experiments, the highest packing density is 0.58 in random loose state, and 0.61 in random dense state of steel balls, poured into a copper cylinder. $\mathrm{He}$ established also, these values decrease with less filling height [7].

The packing density (or porosity) strongly depends on many factors. Basically the geometry and size of particles and reservoir have strong influence on the packing density [8]. Mechanical behaviour of the granulated solid and the pouring method also affect porosity of packed spherical particles [9].

With continuous development of numerical methods more and more possibilities were issued for analysing the particle packing phenomenon. Detailed evaluation on particle shape effect was made by Tangri et al. [10], who founded significant influence of container height and falling velocity of cylindrical particles on particle packing density. Particle-fluid interaction can be also modelled numerically, as it was shown in work of Dong et al. [11].

\section{Numerical modelling}

During this work, DEM was applied for describing particle motion in the snowstorm filler device, which is an iterative procedure to follow motion and interactions of individual particles and walls. Main idea of the numerical approximation is the continuous utilization of Newton's 2nd law of motion and the general rotation equation for calculating displacements of each element, through series of small timesteps [12]. The method can effectively apply for describing particle motion in equipment used in several fields of industry or for optimizing procedures related to granular matters.
Each simulation introduced in this paper was accomplished by using YADE discrete element framework [13]. One of the essential questions related to discrete numerical simulations is the description of interactions. In this study, the linear-elastic contact model was utilized for the simulations. Two main parameters of this contact model are the normal- and shear stiffness of interactions. The normal stiffness was calculated by using Young's modulus $\left(E_{1}, E_{2}\right)$ and radius $\left(r_{1}, r_{2}\right)$ of the contacting elements [13]:

$$
k_{N}=\frac{2 E_{1} r_{1} E_{2} r_{2}}{E_{1} r_{1}+E_{2} r_{2}} \text {. }
$$

The shear stiffness $\left(k_{S}\right)$ is defined as a certain fraction of normal stiffness. Result of interaction are the displacements of colliding elements ( $\mathbf{u}_{\mathbf{N}}$ normal- and $\mathbf{u}_{\mathbf{S}}$ shear directional), which can determine by using geometrical parameters of the contact. Normal displacement is calculated by the following expression

$$
\mathbf{u}_{\mathrm{N}}=\left|\widehat{\mathbf{C}}_{2}-\widehat{\mathbf{C}}_{\mathbf{1}}\right|-\left|\mathbf{C}_{2}-\mathbf{C}_{\mathbf{1}}\right|,
$$

where $\mathbf{C}_{1}$ and $\mathbf{C}_{2}$ are initial centres of interacting elements, as $\widehat{\mathbf{C}}_{\mathbf{1}}$ and $\widehat{\mathbf{C}}_{\mathbf{2}}$ are the updated state variables during motion of particles. The us shear displacement composed by two parts: the motion of contact plane normal and contact point, and the mutual motion of the contacting spheres. More detailed description of the numerical approximation can be found in [13]

The interaction forces in both directions are defined by mechanical and geometrical parameters:

$$
\begin{gathered}
\mathbf{F}_{\mathbf{N}}=k_{N} \mathbf{u}_{\mathbf{N}}, \\
\mathbf{F}_{\mathbf{S}}=\left\{\begin{array}{l}
\mathbf{F}_{\mathbf{N}} \operatorname{tg} \varphi \text { if }\left|k_{S} \mathbf{u}_{\mathbf{S}}\right|>\left|\mathbf{F}_{\mathbf{N}}\right| \operatorname{tg} \varphi \\
k_{S} \mathbf{u}_{\mathbf{S}}
\end{array}\right.
\end{gathered}
$$

where $\varphi$ is the friction angle between interacting elements. Ensuring numerical stability of DEM simulations, there is a threshold limit of the numerical timestep $\left(\Delta t_{c r}\right)$ in YADE [13]

$$
\Delta t_{\text {crit }}=\frac{2}{\omega_{\max }}=\min _{i}\left(\sqrt{2} ; \sqrt{\frac{m_{i}}{k_{i}}}\right),
$$

where $\omega_{\max }$ is the highest eigenfrequency within the system, $m_{i}$ and $k_{i}$ denotes mass and stiffness of the $i$-th particle and spring respectively [13].

\subsection{DEM model}

Snowstorm filling of a real air dryer unit, used in guided ground transport industry was modelled. Within the dryer unit a bottom cone and a central tube with another inner cone can be found (Fig. 2). Main inner diameter of the reservoir is $176 \mathrm{~mm}$ and it has a height of $230 \mathrm{~mm}$. Above the container, the snowstorm device is placed, which is composed by a conical filler bin, and a distribution rod system below it. The filler bin and the reservoir have the same diameter, and the rods are evenly distributed on a single level, as the levels are eq- 

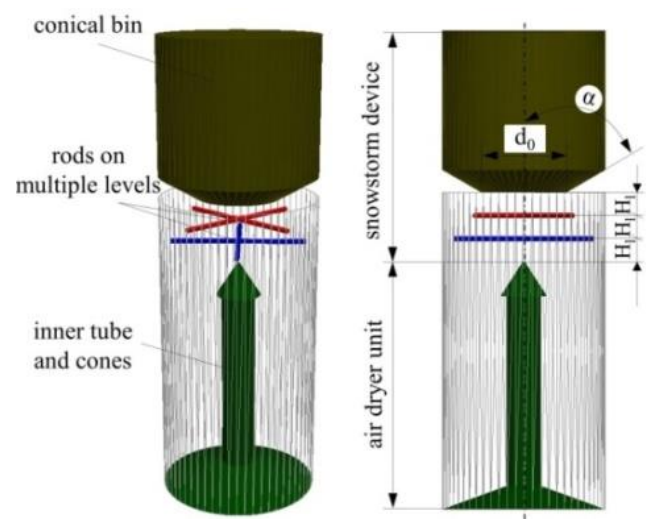

Fig. 2. Geometrical model of the air dryer unit and the snowstorm filler

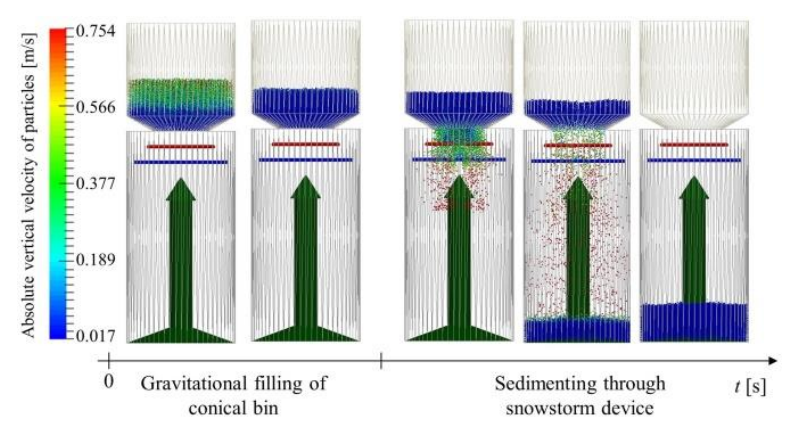

Fig. 3. Steps of DEM simulation

ually spaced below each other (distance between levels is $H_{l}$ ). Cone half angle of the filler bin was $\alpha=10^{\circ}$ and diameter of the outlet was $90 \mathrm{~mm}$. (Fig. 2).

In this specific case sandstone, as a commonly used desiccant material was used in numerical simulations. The particles have almost perfect spherical shape with average diameter of $d_{p}=2 \mathrm{~mm}$ with $0.2 \mathrm{~mm}$ standard deviation. Hence calibration of micromechanical parameters is a key question in each discrete simulation, properties of sandstone particles were identified by accomplishing silo outflow experiments. Detailed description of the calibration procedure and micromechanical parameters of sandstone can be found in our previous work [14].

Steps of simulation are introduced in Fig. 3. whereon the particles were coloured based on their vertical velocity. The elements were randomly generated in a predefined volume within the conical bin, and then were deposited gravitationally in the closed bin. Subsequently it was necessary to wait until the overall kinetic energy of the bulk decreased almost to zero, in other words the system reached quasi static state. The following step is the bin opening, and filling of the air dryer unit.

At the end of the simulation (when the system reached again quasi static state), the total porosity of the deposited assembly was calculated, which is the fraction of the bulk volume, that is not occupied by sandstone particles. During the calculation, the volume filled by spheres $\left(V_{S}\right)$ and the total boundary volume $\left(V_{0}\right)$ was considered according to the following expression

$$
\varepsilon=\frac{\text { overall void volume }}{\text { total boundary volume }}=\frac{V_{0}-V_{S}}{V_{0}}=1-\frac{V_{S}}{V_{0}} .
$$

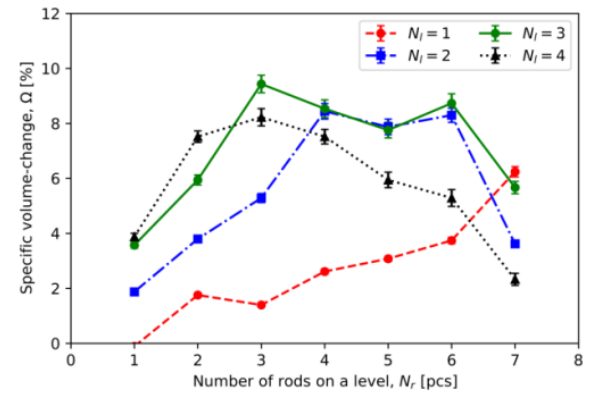

Fig. 4. Specific-volume change versus number of rods on each level, for different arrangements

The boundary volume was determined based on the filling height of the reservoir. Centroid coordinates of the uppermost particle were used for calculating volume of the envelop cylinder. Subtracting total volume of inner tube and cones from this, the total boundary volume $\left(V_{0}\right)$ was obtained.

\subsection{Geometrical optimization}

For finding the geometrical configuration, which ensures highest packing density, the number of rods $\left(N_{r}\right)$ was systematically changed between 1 and 7 , and the number of rod levels in the distribution system $\left(N_{l}\right)$ was also varied in the range $1-4$.

Rod and particle diameter ratio was constant, 2.8 as the vertical distance between these levels was also not changed $\left(H_{l}=25 \mathrm{~mm}\right)$. For each geometrical configuration, the simulations were repeated five times to handle randomness of the phenomenon and average values as well as standard deviations were calculated.

To generalize the results, the specific volumechange $(\Omega)$ were calculated and plotted in function of both $N_{r}$ and $N_{l}$ in Fig. 4. The specific volume-change was determined based on the calculated porosity values, and directly gives information about efficiency improvement of the dryer unit. It is defined as the relative change of total particle volume filled into the reservoir, compared to the spherical volume packed without the snowstorm device $\left(V_{S 0}=1.541 \mathrm{E}-3 \mathrm{~m}^{3}\right)$ :

$$
\Omega=\frac{V_{S}-V_{S 0}}{V_{S 0}} 100 .
$$

As it can be seen in Fig. 4, the specific volume change shows optimum at a certain geometrical configuration. At the optimal arrangement (9 rods evenly distributed on 3 levels), the porosity was decreased by $4.2 \%$ and total volume of the particles filled into the reservoir was improved by $9.5 \%$ (green plot in Fig. 4). This implies that, by applying the optimal snowstorm system, significantly higher packing density can be reached without external excitation.

Laboratory experiments were carried out, for verifying the numerical calculations. The snowstorm filler with 8 rods evenly distributed on 2 levels was 3D printed and used for packing experiments. Based on measured filling height, the experimentally obtained total porosity was calculated. DEM results fit to the observed particle distribution and total porosity, as it can be seen in Fig. 5. Detailed description of the validation 


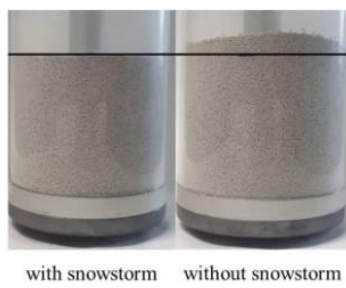

a) experiment

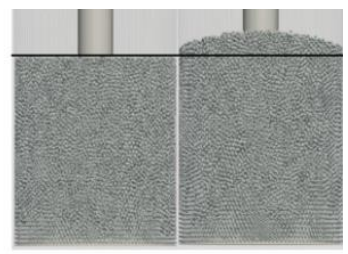

with snowstorm without snowstorm b) DEM
Fig. 5. Experimental and numerical packing of sandstone

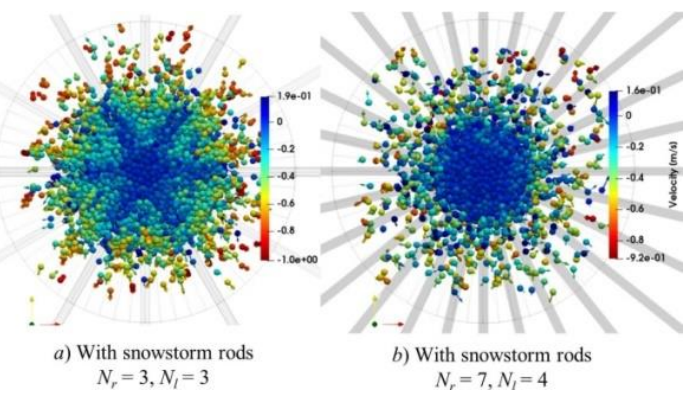

Fig. 6. Vertical particle velocity for different arrangements

process can be found in [14].

Main reason of the packing density improvement can be that, the snowstorm device enhances lateral velocity of particles, which facilitates their even distribution. However, it must consider that, the flowing velocity has direct effect on the porosity, therefore too many obstacles can block the particle stream, which may result looser packing. For deeper analysing the interaction between snowstorm device and particles, the velocity field was calculated (Fig. 6), and the ratio of particles' lateral and vertical velocity components was averaged in certain levels inside the unit (Fig. 7). During the velocity ratio investigation, the optimal snowstorm arrangement (9 rods evenly distributed on 3 levels) was used and vertical distance between distribution levels $\left(H_{l}\right)$ was varied in the range 10 to $40 \mathrm{~mm}$ (compared to the particle diameter, $d_{p}$ the $H_{l}-d_{p}$ ratio was between 5 and 20).

Based on the velocity ratio change shown in Fig. 7, the improvement of lateral velocity component is obvious. Optimal rod level distance was also revealed, as the higher lateral velocity facilitates spread of particles The packing density increases because of even distribution of spheres, but too many interactions lead to considerably high vertical velocity decrease as it is shown in Fig. 6. By applying the ideal distance between the rod levels, the particles' lateral velocity could reach almost $30 \%$ of the vertical velocity component (Fig. 7).

\section{Results and conclusions}

Two opposite effects of the snowstorm unit were recognized based on our study. From one hand, the rods increase lateral velocity of the particles, thus these can uniformly fill the reservoir. Moreover, it was revealed that, the number of rod-particle interactions is strongly affected by the distance between rod levels. On the other hand, the distribution system also works as an obstacle in the particle flow. As the packing density is strongly influenced by the flow flux of particles, the number and arrangement of the rods must be carefully selected, in

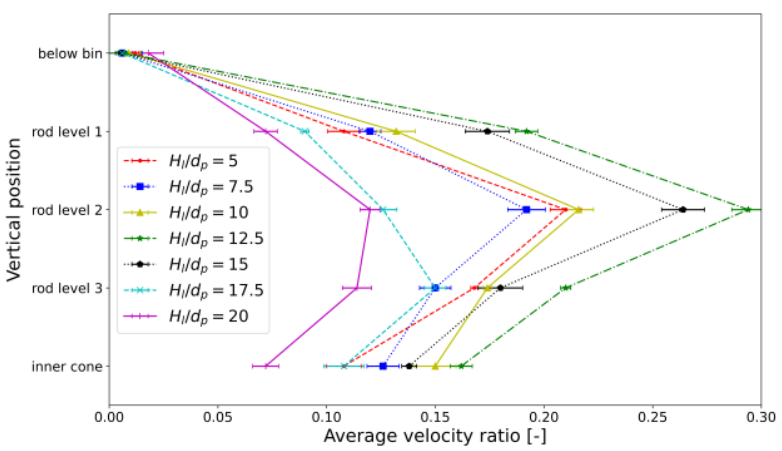

Fig. 7. Average velocity ratio for different level distances order to do not block significantly the particle flow.

By the revealed relationships, the filling procedure of an industrial air dryer unit could be significantly improved without applying external energy. With the suggested snowstorm unit arrangement, by $9.5 \%$ more sandstone particles can be settled into the reservoir, compared to the usual industrial filling procedure. Not only the energy consumption can be minimized in this way, but both operational and maintenance costs can be decreased, compared to commonly used shakers.

Our work was supported by the ÚNKP-20-4 New National Excellence Program of the Ministry for Innovation and Technology from the source of the National Research, Development and Innovation Fund.

\section{References}

1. E. Harris, Improvement in or relating to the filling of granular materials into container, patent number GB606867A, United Kingdom, 1946-0121.

2. C. Gonzalez-Montanello, A. Ramírez, E. Gallego, F. Ayuga, Chem. Eng. Sci. 66, 21 (2011)

3. I. Oldal, F. Safranyik, J. Mech. Sci. Tech. 29, 9 (2015)

4. I. Oldal, I. Keppler, B. Csizmadia, L. Fenyvesi, Adv. Pow. Tech. 23, (2012)

5. M.C.V. Olivares, J.G. Benito, R.O. Unac, A.M. Vidales, Pow. Tech. 336 (2018)

6. I. Zuriguel, A. Garcimartín, D. Maza, L.A. Pugnaloni, J.M. Pastor, Phys. Rev. E 71, (2005)

7. G.D. Scott, Nature 188, (1960)

8. R.P. Zou, A.B. You, Chem. Eng. Sci. 5, (1995)

9. J.C. Macrae, W.A. Gray, Br. J. Appl. Phys. 12, (1960)

10. H. Tangri, Y. Guoa, J.S. Curtis, Powd. Tech. 317, (2017)

11. K.J. Dong, R.Y. Yang, R.P. Zou, A.B. Yu, AIChE J. 58, 5 (2012)

12. P.A. Cundall, O.D.L. Strack, Geotech. 29, (1979)

13. V. Šmilauer, B. Chareyre, Yade dem formulation. In Yade Documentation (V. Šmilauer, ed.), The Yade Project, 1st ed., (2010)

14. F. Safranyik, A. Varga, I. Oldal, I. Keppler, Adv. Pow. Tech. 31, 8 (2020) 